\title{
Some New Dynamic Inequalities Involving Monotonic Functions on Time Scales
}

\author{
S. H. Saker $\mathbb{D}^{1}{ }^{1}$ E. Awwad, ${ }^{2,3}$ and A. Saied ${ }^{3}$ \\ ${ }^{1}$ Department of Mathematics, Faculty of Science, Mansoura University, Mansoura, Egypt \\ ${ }^{2}$ Department of Mathematics, College of Science and Arts, Gurayat, Jouf University, Saudi Arabia \\ ${ }^{3}$ Department of Mathematics, Faculty of Science, Benha University, Benha, Egypt
}

Correspondence should be addressed to S. H. Saker; shsaker@mans.edu.eg

Received 25 March 2019; Accepted 24 April 2019; Published 8 May 2019

Academic Editor: Gestur Ólafsson

Copyright (C) 2019 S. H. Saker et al. This is an open access article distributed under the Creative Commons Attribution License, which permits unrestricted use, distribution, and reproduction in any medium, provided the original work is properly cited.

In this paper, we prove some new dynamic inequalities involving $C$ - monotonic functions on time scales. The main results will be proved by employing Hölder's inequality, integration by parts, and a chain rule on time scales. As a special case when $\mathbb{T}=\mathbb{R}$, our results contain the continuous inequalities proved by Heinig, Maligranda, Pečarić, Perić, and Persson and when $\mathbb{T}=\mathbb{N}$, the results to the best of the authors' knowledge are essentially new.

\section{Introduction}

In 1995 Heinig and Maligranda [1] proved that if $-\infty \leq a<$ $b \leq \infty, f, g \geq 0, f$ is decreasing on $(a, b)$ and $g$ is increasing on $(a, b)$ with $g(a)=0$, then for any $\gamma \in(0,1]$

$$
\int_{a}^{b} f(x) d g(x) \leq\left(\int_{a}^{b} f^{\gamma}(x) d\left[g^{\gamma}(x)\right]\right)^{1 / \gamma}
$$

Inequality (1) is reversed when $1 \leq \gamma<\infty$. Also in [1] they proved that if $f$ is increasing on $(a, b)$ and $g$ is decreasing on $(a, b)$ with $g(b)=0$, then, for any $\gamma \in(0,1]$,

$$
\int_{a}^{b} f(x) d[-g(x)] \leq\left(\int_{a}^{b} f^{\gamma}(x) d\left[-g^{\gamma}(x)\right]\right)^{1 / \gamma}
$$

In [1] the authors generalized (1) and proved that if $0<p \leq$ $q<\infty$, and $u, v$ are positive functions, then there exists a constant $D>0$ such that the inequality,

$$
\begin{aligned}
& {\left[\int_{0}^{\infty} u(x) f^{q}(x) d x\right]^{1 / q}} \\
& \quad \leq D\left[\int_{0}^{\infty} v(x) f^{p}(x) d x\right]^{1 / p},
\end{aligned}
$$

holds for all nonnegative decreasing function $f$ if and only if

$$
\left[\int_{0}^{t} u(x) d x\right]^{1 / q} \leq D\left[\int_{0}^{t} v(x) d x\right]^{1 / p}, \quad \forall t>0 .
$$

In [1] it is also proved that inequality (3) holds for all nonnegative increasing functions $f$ and $0<p \leq q<\infty$ if and only if

$$
\left[\int_{t}^{\infty} u(x) d x\right]^{1 / q} \leq D\left[\int_{t}^{\infty} v(x) d x\right]^{1 / p}, \quad \forall t>0 .
$$

In 1997 Pečarić et al. [2] generalized (1) and proved that if $f$ is $C$-decreasing with $C \geq 1$ and $g$ is an increasing function on $[a, b]$ such that $g(a)=0$, and $\varphi:[0, \infty) \longrightarrow \mathbb{R}$ is a concave, nonnegative, and differentiable function such that $\varphi(0)=0$, then

$$
\begin{aligned}
& \varphi\left(C \int_{a}^{b} f(x) d g(x)\right) \\
& \quad \leq C \int_{a}^{b} \varphi^{\prime}[f(x) g(x)](f(x) d g(x)) .
\end{aligned}
$$

The function $f$ is said to be $C$-decreasing if $s \leq t$ implies that $f(t) \leq C f(s)$ for $C>1$. Pečarić et al. [2] also proved that if $f$ 
is $C$-increasing with $C \geq 1$ and $g$ is increasing on $[a, b]$, such that $g(a)=0$, then

$$
\begin{aligned}
& \varphi\left(\frac{1}{C} \int_{a}^{b} f(x) d g(x)\right) \\
& \quad \geq \frac{1}{C} \int_{a}^{b} \varphi^{\prime}[f(x) g(x)] f(x) d g(x) .
\end{aligned}
$$

The function $f$ is said to be $C$-increasing if $s \leq t$ implies $f(s) \leq C f(t)$. Furthermore they also considered the case when $f$ is $C$ - increasing with $C \geq 1$ and $g$ is decreasing on $[a, b]$, such that $g(b)=0$, and proved that

$$
\begin{aligned}
& \varphi\left(C \int_{a}^{b} f(x) d[-g(x)]\right) \\
& \quad \leq C \int_{a}^{b} \varphi^{\prime}[f(x) g(x)] f(x) d[-g(x)] .
\end{aligned}
$$

Finally they proved that if $f$ is $C$-decreasing with $C \geq 1$ and $g$ is decreasing on $[a, b]$, such that $g(b)=0$, then

$$
\begin{aligned}
\varphi( & \left.\frac{1}{C} \int_{a}^{b} f(x) d[-g(x)]\right) \\
& \geq \frac{1}{C} \int_{a}^{b} \varphi^{\prime}[f(x) g(x)] f(x) d[-g(x)] .
\end{aligned}
$$

In the last decades some authors have been interested in finding some discrete results on $l^{p}(\mathbb{N})$ analogues to $L^{p}(\mathbb{R})$ bounds in different fields in analysis and as a result this subject becomes a topic of ongoing research. One reason for this upsurge of interest in discrete case is also due to the fact that discrete operators may even behave differently from their continuous counterparts. In this paper, we obtain the discrete inequalities as special cases of the results with a general domain called the time scale $\mathbb{T}$, which is an arbitrary nonempty closed subset of the real numbers $\mathbb{R}$. These new results on the time scale $\mathbb{T}$ contain the classical continuous and discrete inequalities as special cases when $\mathbb{T}=\mathbb{R}$ and $\mathbb{T}=\mathbb{N}$ and can be extended to different inequalities on different time scales such as $\mathbb{T}=h \mathbb{N}, h>0, \mathbb{T}=q^{\mathbb{N}}$ for $q>1$.

In recent years the study of dynamic inequalities on time scales has received a lot of attention and has become a major field in pure and applied mathematics. For more details about the dynamic inequalities on time scales, we refer the reader to the books [3-5] and the papers [6-11].

The natural question now is the following: Is it possible to prove some new inequalities with $C$-monotonic $r d$-continuous functions defined on a time scale $\mathbb{T}$ and as special cases contain the above results?

Our aim in this paper is to give the answer to this question and find the relation between the weighted functions which ensure that the inequality

$$
\begin{aligned}
& {\left[\int_{a}^{\infty} w(x) f^{q}(x) \Delta x\right]^{1 / q}} \\
& \quad \leq D\left[\int_{a}^{\infty} h(x) f^{p}(x) \Delta x\right]^{1 / p},
\end{aligned}
$$

holds for all nonnegative decreasing function $f$ such that $\lim _{x \rightarrow \infty} f(x)=0, D>0$, and $0<p \leq q<\infty$. Also, we establish some new dynamic inequalities involving $C$-monotonic functions in the form

$$
\begin{aligned}
& \varphi\left(C \int_{a}^{b} f(x) g^{\Delta}(x) \Delta x\right) \\
& \quad \leq C \int_{a}^{b} f(x) g^{\Delta}(x) \varphi^{\prime}[f(x) g(x)] \Delta x,
\end{aligned}
$$

where $\varphi:[0, \infty) \longrightarrow \mathbb{R}$ is a concave, nonnegative, and differentiable function such that $\varphi(0)=0$, and $f$ is $C$ decreasing with $C \geq 1$, and $g$ is an increasing function on $[a, b]_{\mathbb{T}}$, such that $g(a)=0$.

The paper is organized as follows. In Section 2, we present some preliminaries concerning the theory of time scales and prove the basic lemmas that will be needed in the proofs. In Section 3, we prove the main results by using Hölder's inequality, integration by parts, and a chain rule on time scales. Our results when $\mathbb{T}=\mathbb{R}$ give inequalities (1), (2), (3), (6), (7), (8), and (9) proved by Heinig, Maligranda, Pečarić, Perić, and Persson. When $\mathbb{T}=\mathbb{N}$, our results are essentially new.

\section{Preliminaries and Basic Lemmas}

In this section, we recall the following concepts related to the notion of time scales. For more details of time scale analysis we refer the reader to the two books by Bohner and Peterson $[5,12]$ which summarize and organize much of the time scale calculus. A time scale $\mathbb{T}$ is an arbitrary nonempty closed subset of the real numbers $\mathbb{R}$. The forward jump operator and the backward jump operator are defined by $\sigma(t):=\inf \{s \in \mathbb{T}$ : $s>t\}$, and $\rho(t):=\sup \{s \in \mathbb{T}: s<t\}$, where $\sup \emptyset=\inf \mathbb{T}$. A point, $t \in \mathbb{T}$, is said to be left-dense if $\rho(t)=t$ and $t>\inf \mathbb{T}$, is right-dense if $\sigma(t)=t$, is left-scattered if $\rho(t)<t$, and is right-scattered if $\sigma(t)>t$. A function $f: \mathbb{T} \longrightarrow \mathbb{R}$ is said to be right-dense continuous (rd-continuous) provided $f$ is continuous at right dense points and, at left-dense points in $\mathbb{T}$, left hand limits exist and are finite. The set of all such rdcontinuous functions is denoted by $C_{r d}(\mathbb{T})=C_{r d}(\mathbb{T}, \mathbb{R})$. The product and quotient rules for the derivative of the product $f g$ and the quotient $f / g$ (where $g g^{\sigma} \neq 0$, here $g^{\sigma}=g \circ \sigma$ ) of two differentiable functions $f$ and $g$ are given by

$$
\begin{aligned}
& (f g)^{\Delta}=f g^{\Delta}+f^{\Delta} g^{\sigma}=f^{\Delta} g+f^{\sigma} g^{\Delta}, \\
& \left(\frac{f}{g}\right)^{\Delta}=\frac{f^{\Delta} g-f g^{\Delta}}{g g^{\sigma}} .
\end{aligned}
$$

Let $f: \mathbb{R} \longrightarrow \mathbb{R}$ be continuously differentiable and suppose that $g: \mathbb{T} \longrightarrow \mathbb{R}$ is delta differentiable. Then $f \circ g: \mathbb{T} \longrightarrow \mathbb{R}$ is delta differentiable and there exists $d$ in the real interval $[t, \sigma(t)]$ for $t \in \mathbb{T}$, such that

$$
(f \circ g)^{\Delta}(t)=f^{\prime}(g(d)) g^{\Delta}(t) .
$$


Another shape of the chain rule is the formula

$$
\begin{aligned}
& (f \circ g)^{\Delta}(t) \\
& \quad=\left\{\int_{0}^{1} f^{\prime}\left(g(t)+h \mu(t) g^{\Delta}(t)\right) d h\right\} g^{\Delta}(t) .
\end{aligned}
$$

A special case of (14) is

$$
\left[u^{\lambda}(t)\right]^{\Delta}=\lambda \int_{0}^{1}\left[h u^{\sigma}+(1-h) u\right]^{\lambda-1} u^{\Delta}(t) d h .
$$

We define the time scale interval $[a, b]_{\mathbb{T}}$ by $[a, b]_{\mathbb{T}}:=[a, b] \cap$ $\mathbb{T}$. In this paper, we will use Cauchy (delta) integral which we can define as follows. If $G^{\Delta}(t)=g(t)$, then the Cauchy (delta) integral of $g$ is defined by $\int_{a}^{t} g(x) \Delta x:=G(t)-G(a)$. It can be shown (see [5]) that if $g \in C_{r d}(\mathbb{\mathbb { T }})$, then the Cauchy integral $G(t):=\int_{t_{0}}^{t} g(x) \Delta x$ exists, $t_{0} \in \mathbb{T}$, and satisfies $G^{\Delta}(t)=$ $g(t), t \in \mathbb{T}$. In case $\mathbb{T}=\mathbb{R}$, we have

$$
\begin{aligned}
\sigma(t) & =\rho(t)=t, \\
\mu(t) & =0, \\
f^{\Delta} & =f^{\prime}, \\
\int_{a}^{b} f(t) \Delta t & =\int_{a}^{b} f(t) d t,
\end{aligned}
$$

The integration on discrete time scales is defined by $\int_{a}^{b} g(t) \Delta t=\sum_{t \in[a, b)} \mu(t) g(t)$ and then, in case $\mathbb{T}=\mathbb{Z}$, we have

$$
\begin{aligned}
\sigma(t) & =t+1, \\
\rho(t) & =t-1, \\
\mu(t) & =1, \\
f^{\Delta} & =\Delta f \\
\int_{a}^{b} f(t) \Delta t & =\sum_{t=a}^{b-1} f(t) .
\end{aligned}
$$

The integration by parts formula on time scales is given by

$$
\int_{a}^{b} u^{\Delta}(t) v^{\sigma}(t) \Delta t=\left.u(t) v(t)\right|_{a} ^{b}-\int_{a}^{b} u(t) v^{\Delta}(t) \Delta t
$$

Also, we have, for $f \in C_{r d}$ and $t \in \mathbb{T}$, that

$$
\int_{t}^{\sigma(t)} f(\tau) \Delta \tau=\mu(t) f(t) .
$$

The Hölder inequality on time scales is given by

$$
\begin{aligned}
& \int_{a}^{b} f(t) g(t) \Delta t \\
& \quad \leq\left(\int_{a}^{b} f^{\gamma}(t) \Delta t\right)^{1 / \gamma}\left(\int_{a}^{b} g^{\nu}(t) \Delta t\right)^{1 / \nu},
\end{aligned}
$$

where $>1,1 / \gamma+1 / \nu=1$, and $f, g \in C_{r d}\left([a, b]_{\mathbb{T}}, \mathbb{R}^{+}\right)$. Inequality (20) is reversed if $0<\gamma<1$ or $\gamma<0$.
Definition 1. A set $K \subset \mathbb{R}$ is convex if, for all $x, y \in K$ and $\lambda \in[0,1]$, we have

$$
\lambda x+(1-\lambda) y \in K .
$$

A function $F: K \subset \mathbb{R} \longrightarrow \mathbb{R}$ is concave if $K$ is convex and, for all $x, y \in K$ and $\lambda \in[0,1]$, we have

$$
F(\lambda x+(1-\lambda) y) \geq \lambda F(x)+(1-\lambda) F(y) .
$$

A function $F: K \subset \mathbb{R} \longrightarrow \mathbb{R}$ is convex if $-F$ is concave.

Definition 2. Assume that $\mathbb{T}$ is a time scale, $f: \mathbb{T} \longrightarrow \mathbb{R}$ and $C \geq 1$. If $s \leq t$ implies $f(t) \leq C f(s)$, then $f$ is $C$-decreasing. If $s \leq t$ implies $f(s) \leq C f(t)$, then $f$ is $C$-increasing. As a special case when $C=1$ we get the classical definitions.

Now, we prove the basic lemmas that will be used to prove our main results. Throughout the paper, we assume that the functions (without mentioning) are rd-continuous nonnegative and $\Delta$-differentiable functions, locally $\Delta$-integrable on $[a, \infty)_{\mathbb{T}}$ and the integrals considered are assumed to exist and finite.

Lemma 3. Assume that $\mathbb{T}$ is a time scale with $a \in \mathbb{T}$ and, $\beta \in C_{r d}\left([a, \infty)_{\mathbb{T}}, \mathbb{R}^{+}\right)$. If $\gamma \geq 1$, then

$$
\begin{gathered}
{\left[\int_{a}^{\infty} \alpha(x)\left(\int_{a}^{\sigma(x)} \beta(\tau) \Delta \tau\right)^{\gamma} \Delta x\right]^{1 / \gamma}} \\
\quad \leq \int_{a}^{\infty} \beta(x)\left(\int_{x}^{\infty} \alpha(s) \Delta s\right)^{1 / \gamma} \Delta x .
\end{gathered}
$$

Proof. Let $F(x)=\int_{a}^{x} \beta(\tau) \Delta \tau$. Then, the left hand side of (23) can be written in the form

$$
\begin{aligned}
\int_{a}^{\infty} \alpha(x)\left(\int_{a}^{\sigma(x)} \beta(\tau) \Delta \tau\right)^{\gamma} \Delta x \\
=\int_{a}^{\infty} \alpha(x)\left[F^{\sigma}(x)\right]^{\gamma-1} F^{\sigma}(x) \Delta x .
\end{aligned}
$$

Integrating the right hand side of (24) by parts with

$$
\begin{aligned}
u^{\Delta}(x) & =\alpha(x)\left[F^{\sigma}(x)\right]^{\gamma-1}, \\
\nu^{\sigma}(x) & =F^{\sigma}(x),
\end{aligned}
$$

we have that

$$
\begin{aligned}
\int_{a}^{\infty} & \alpha(x)\left[F^{\sigma}(x)\right]^{\gamma-1} F^{\sigma}(x) \Delta x \\
& =\left.u(x) F(x)\right|_{a} ^{\infty}-\int_{a}^{\infty} u(x) \beta(x) \Delta x,
\end{aligned}
$$

where $u(x)=-\int_{x}^{\infty} \alpha(s)\left[F^{\sigma}(s)\right]^{\gamma-1} \Delta s$. Using the facts that $\lim _{x \rightarrow \infty} u(x)=0$ and $F(a)=0$, we see that

$$
\begin{aligned}
\int_{a}^{\infty} & \alpha(x)\left[F^{\sigma}(x)\right]^{\gamma-1} F^{\sigma}(x) \Delta x \\
& =\int_{a}^{\infty} \beta(x)\left(\int_{x}^{\infty} \alpha(s)\left[F^{\sigma}(s)\right]^{\gamma-1} \Delta s\right) \Delta x .
\end{aligned}
$$


Substituting (27) into (24), we get that

$$
\begin{gathered}
\int_{a}^{\infty} \alpha(x)\left(\int_{a}^{\sigma(x)} \beta(\tau) \Delta \tau\right)^{\gamma} \Delta x=\int_{a}^{\infty} \beta(x) \\
\cdot\left(\int_{x}^{\infty} \alpha(s)\left[F^{\sigma}(s)\right]^{\gamma-1} \Delta s\right) \Delta x=\int_{a}^{\infty} \beta(x) \\
\cdot\left(\int_{x}^{\infty} \alpha^{1 / \gamma}(s) \alpha^{(\gamma-1) / \gamma}(s)\left[F^{\sigma}(s)\right]^{\gamma-1} \Delta s\right) \Delta x .
\end{gathered}
$$

Applying the Hölder inequality with indices $\gamma>1$, and $\gamma /(\gamma-$ 1) on the term

$$
\int_{x}^{\infty} \alpha^{1 / \gamma}(s) \alpha^{(\gamma-1) / \gamma}(s)\left[F^{\sigma}(s)\right]^{\gamma-1} \Delta s
$$

we see that

$$
\begin{aligned}
& \int_{x}^{\infty} \alpha^{1 / \gamma}(s) \alpha^{(\gamma-1) / \gamma}(s)\left[F^{\sigma}(s)\right]^{\gamma-1} \Delta s \\
& \leq\left(\int_{x}^{\infty} \alpha(s) \Delta s\right)^{1 / \gamma}\left(\int_{x}^{\infty} \alpha(s)\left[F^{\sigma}(s)\right]^{\gamma} \Delta s\right)^{(\gamma-1) / \gamma} .
\end{aligned}
$$

Substituting (30) into (28), where $\beta$ is a positive function, we obtain

$$
\begin{gathered}
\int_{a}^{\infty} \alpha(x)\left(\int_{a}^{\sigma(x)} \beta(\tau) \Delta \tau\right)^{\gamma} \Delta x \leq \int_{a}^{\infty} \beta(x) \\
\cdot\left(\int_{x}^{\infty} \alpha(s) \Delta s\right)^{1 / \gamma} \\
\cdot\left(\int_{x}^{\infty} \alpha(s)\left[F^{\sigma}(s)\right]^{\gamma} \Delta s\right)^{(\gamma-1) / \gamma} \Delta x .
\end{gathered}
$$

Since $x \geq a$ and $\alpha, \beta$ are positive functions, we see that

$$
\int_{x}^{\infty} \alpha(s)\left[F^{\sigma}(s)\right]^{\gamma} \Delta s \leq \int_{a}^{\infty} \alpha(s)\left[F^{\sigma}(s)\right]^{\gamma} \Delta s
$$

and then we have for $\gamma>1$ (note that $(\gamma-1) / \gamma>0$ ) that

$$
\begin{aligned}
& \left(\int_{x}^{\infty} \alpha(s)\left[F^{\sigma}(s)\right]^{\gamma} \Delta s\right)^{(\gamma-1) / \gamma} \\
& \quad \leq\left(\int_{a}^{\infty} \alpha(s)\left[F^{\sigma}(s)\right]^{\gamma} \Delta s\right)^{(\gamma-1) / \gamma} .
\end{aligned}
$$

From (31) and (33), we have

$$
\begin{aligned}
& \int_{a}^{\infty} \alpha(x)\left(\int_{a}^{\sigma(x)} \beta(\tau) \Delta \tau\right)^{\gamma} \Delta x \\
& \leq\left(\int_{a}^{\infty} \alpha(s)\left[F^{\sigma}(s)\right]^{\gamma} \Delta s\right)^{(\gamma-1) / \gamma} \\
& \cdot \int_{a}^{\infty} \beta(x)\left(\int_{x}^{\infty} \alpha(s) \Delta s\right)^{1 / \gamma} \Delta x \\
& =\left[\int_{a}^{\infty} \alpha(s)\left(\int_{a}^{\sigma(s)} \beta(\tau) \Delta \tau\right)^{\gamma} \Delta s\right]^{(\gamma-1) / \gamma} \\
& \cdot \int_{a}^{\infty} \beta(x)\left(\int_{x}^{\infty} \alpha(s) \Delta s\right)^{1 / \gamma} \Delta x,
\end{aligned}
$$

and then

$$
\begin{gathered}
{\left[\int_{a}^{\infty} \alpha(x)\left(\int_{a}^{\sigma(x)} \beta(\tau) \Delta \tau\right)^{\gamma} \Delta x\right]^{1 / \gamma}} \\
\quad \leq \int_{a}^{\infty} \beta(x)\left(\int_{x}^{\infty} \alpha(s) \Delta s\right)^{1 / \gamma} \Delta x
\end{gathered}
$$

which is the desired inequality (23). The proof is complete.

As in the proof of Lemma 3, we can easily prove the following dual lemma.

Lemma 4. Assume that $\mathbb{T}$ is a time scale with $a \in \mathbb{T}$ and, $\beta \in C_{r d}\left([a, \infty)_{\mathbb{T}}, \mathbb{R}^{+}\right)$. If $\gamma \geq 1$, then

$$
\begin{aligned}
& {\left[\int_{a}^{\infty} \alpha(x)\left(\int_{x}^{\infty} \beta(\tau) \Delta \tau\right)^{\gamma} \Delta x\right]^{1 / \gamma}} \\
& \quad \leq \int_{a}^{\infty} \beta(x)\left(\int_{a}^{\sigma(x)} \alpha(s) \Delta s\right)^{1 / \gamma} \Delta x .
\end{aligned}
$$

\section{Main Results}

In this section, we state and prove our main results and for simplicity, we will assume that $w, h$ are positive rd-continuous functions on $[a, \infty)_{\mathbb{T}}$ and $\varphi:[0, \infty) \longrightarrow[0, \infty)$ is a concave and differentiable function such that $\varphi(0)=0$. We begin with the time scale version of (3).

Theorem 5. Assume that $\mathbb{T}$ is a time scale with $a \in \mathbb{T}, 0<$ $p \leq q<\infty$. Furthermore assume that $f$ is nonnegative and decreasing function such that $\lim _{s \rightarrow \infty} f(s)=0$ and

$$
\int_{a}^{\infty} h(x) f^{p}(x) \Delta x<\infty .
$$

If there exists a constant $D>0$ such that

$$
\begin{array}{r}
\left(\int_{a}^{\sigma(x)} w(\tau) \Delta \tau\right)^{1 / q} \leq D\left(\int_{a}^{\sigma(x)} h(\tau) \Delta \tau\right)^{1 / p}, \\
\forall x \in[a, \infty)_{\mathbb{T}},
\end{array}
$$


then

$$
\begin{aligned}
& {\left[\int_{a}^{\infty} w(x) f^{q}(x) \Delta x\right]^{1 / q}} \\
& \quad \leq D\left[\int_{a}^{\infty} h(x) f^{p}(x) \Delta x\right]^{1 / p} .
\end{aligned}
$$

Proof. Integrating the term

$$
\int_{a}^{\infty} w(x) f^{q}(x) \Delta x
$$

by parts formula with $u(x)=f^{q}(x)$ and $v^{\Delta}(x)=w(x)$, we have that

$$
\begin{aligned}
\int_{a}^{\infty} w(x) f^{q}(x) \Delta x= & \left.f^{q}(x) v(x)\right|_{a} ^{\infty} \\
& -\int_{a}^{\infty}\left[f^{q}(x)\right]^{\Delta} v^{\sigma}(x) \Delta x
\end{aligned}
$$

where $v(x)=\int_{a}^{x} w(\tau) \Delta \tau$. Using the facts that $v(a)=0$ and $\lim _{x \rightarrow \infty} f(x)=0$, we obtain

$$
\begin{gathered}
\int_{a}^{\infty} w(x) f^{q}(x) \Delta x=\int_{a}^{\infty}\left[-f^{q}(x)\right]^{\Delta} v^{\sigma}(x) \Delta x \\
=\int_{a}^{\infty}\left[-f^{q}(x)\right]^{\Delta}\left(\int_{a}^{\sigma(x)} w(\tau) \Delta \tau\right) \Delta x,
\end{gathered}
$$

and then

$$
\begin{aligned}
& \left(\int_{a}^{\infty} w(x) f^{q}(x) \Delta x\right)^{p / q} \\
& \quad=\left[\int_{a}^{\infty}\left[-f^{q}(x)\right]^{\Delta}\left(\int_{a}^{\sigma(x)} w(\tau) \Delta \tau\right) \Delta x\right]^{p / q} .
\end{aligned}
$$

Substituting (38) into (43), we see (note $f$ is decreasing) that

$$
\begin{aligned}
& \left(\int_{a}^{\infty} w(x) f^{q}(x) \Delta x\right)^{p / q} \\
& \leq D^{p}\left[\int_{a}^{\infty}\left[-f^{q}(x)\right]^{\Delta}\left(\int_{a}^{\sigma(x)} h(\tau) \Delta \tau\right)^{q / p} \Delta x\right]^{p / q} .
\end{aligned}
$$

Applying (23) with $\gamma=q / p, \alpha(x)=\left[-f^{q}(x)\right]^{\Delta}$ and $\beta(\tau)=$ $h(\tau)$ on the right side of $(44)$, we see that

$$
\begin{gathered}
{\left[\int_{a}^{\infty}\left[-f^{q}(x)\right]^{\Delta}\left(\int_{a}^{\sigma(x)} h(\tau) \Delta \tau\right)^{q / p} \Delta x\right]^{p / q}} \\
\leq \int_{a}^{\infty} h(x)\left(\int_{x}^{\infty}\left[-f^{q}(s)\right]^{\Delta} \Delta s\right)^{p / q} \Delta x .
\end{gathered}
$$

Using the assumption that $\lim _{x \rightarrow \infty} f(x)=0$, we get

$$
\begin{aligned}
& {\left[\int_{a}^{\infty}\left[-f^{q}(x)\right]^{\Delta}\left(\int_{a}^{\sigma(x)} h(\tau) \Delta \tau\right)^{q / p} \Delta x\right]^{p / q}} \\
& \quad \leq \int_{a}^{\infty} h(x) f^{p}(x) \Delta x .
\end{aligned}
$$

Substituting (46) into (44), we have

$$
\left(\int_{a}^{\infty} w(x) f^{q}(x) \Delta x\right)^{p / q} \leq D^{p} \int_{a}^{\infty} h(x) f^{p}(x) \Delta x,
$$

and then

$$
\begin{aligned}
& \left(\int_{a}^{\infty} w(x) f^{q}(x) \Delta x\right)^{1 / q} \\
& \quad \leq D\left(\int_{a}^{\infty} h(x) f^{p}(x) \Delta x\right)^{1 / p},
\end{aligned}
$$

which is the desired inequality (39). The proof is complete.

Remark 6. Suppose that the inequality

$$
\begin{aligned}
& {\left[\int_{a}^{\infty} w(x) f^{q}(x) \Delta x\right]^{1 / q}} \\
& \quad \leq D\left[\int_{a}^{\infty} h(x) f^{p}(x) \Delta x\right]^{1 / p},
\end{aligned}
$$

holds for all nonnegative decreasing functions $f$. Then it holds when

$$
f(x)= \begin{cases}1, & \text { if } x \in[a, \sigma(t)]_{\mathbb{T}}, \\ 0, & \text { if } x \notin[a, \sigma(t)]_{\mathbb{T}}\end{cases}
$$

for any fixed number $t \in[a, \infty)_{\mathbb{T}}$ and becomes

$$
\left[\int_{a}^{\sigma(t)} w(x) \Delta x\right]^{1 / q} \leq D\left[\int_{a}^{\sigma(t)} h(x) \Delta x\right]^{1 / p}
$$

This proves the necessary condition of Theorem 5 .

From Theorem 5 and Remark 6, we have the following corollary.

Corollary 7. Assume that $\mathbb{T}$ is a time scale with $a \in \mathbb{T}, 0<$ $p \leq q<\infty$. If $f$ is nonnegative and decreasing function such that $\lim _{s \longrightarrow \infty} f(s)=0$, and

$$
\int_{a}^{\infty} h(x) f^{p}(x) \Delta x<\infty
$$

then there exists a constant $D>0$ such that the inequality

$$
\begin{aligned}
& {\left[\int_{a}^{\infty} w(x) f^{q}(x) \Delta x\right]^{1 / q}} \\
& \quad \leq D\left[\int_{a}^{\infty} h(x) f^{p}(x) \Delta x\right]^{1 / p},
\end{aligned}
$$

holds if and only if

$$
\left(\int_{a}^{\sigma(x)} w(\tau) \Delta \tau\right)^{1 / q} \leq D\left(\int_{a}^{\sigma(x)} h(\tau) \Delta \tau\right)^{1 / p},
$$

$$
\forall x \in[a, \infty)_{\mathbb{T}} .
$$


Remark 8. As a special case of Corollary 7 when $\mathbb{T}=\mathbb{R}$, we get the integral inequality (3) proved by Heinig and Maligranda [1].

Remark 9. As a special case of Corollary 7 when $\mathbb{T}=\mathbb{N}$ and $a=1$, we see that the inequality

$$
\left[\sum_{n=1}^{\infty} w_{n} f_{n}^{q}\right]^{1 / q} \leq D\left[\sum_{n=1}^{\infty} h_{n} f_{n}^{p}\right]^{1 / p}
$$

holds if and only if

$$
\left(\sum_{k=1}^{n} w_{k}\right)^{1 / q} \leq D\left(\sum_{k=1}^{n} h_{k}\right)^{1 / p}, \quad \forall n \in \mathbb{N},
$$

and $0<p \leq q<\infty$, for nonnegative and decreasing sequences $f$ when $\lim _{n \rightarrow \infty} f(n)=0$.

Theorem 10. Assume that $\mathbb{\mathbb { T }}$ is a time scale with $a \in \mathbb{T}, 0<p \leq$ $q<\infty$. Furthermore assume that $f$ is a nonnegative bounded and increasing function such that $f(a)=0$ and

$$
\int_{a}^{\infty} h(x)\left[f^{\sigma}(x)\right]^{p} \Delta x<\infty .
$$

If there exists a constant $D>0$ such that

$$
\left[\int_{x}^{\infty} w(\tau) \Delta \tau\right]^{1 / q} \leq D\left[\int_{x}^{\infty} h(\tau) \Delta \tau\right]^{1 / p}
$$

$$
\forall x \in[a, \infty)_{\mathbb{T}},
$$

then

$$
\begin{aligned}
& {\left[\int_{a}^{\infty} w(x) f^{q}(x) \Delta x\right]^{1 / q}} \\
& \quad \leq D\left[\int_{a}^{\infty} h(x)\left[f^{\sigma}(x)\right]^{p} \Delta x\right]^{1 / p} .
\end{aligned}
$$

Proof. Applying the integration by parts on the term $\int_{a}^{\infty} w(x) f^{q}(x) \Delta x$ with $u(x)=f^{q}(x)$ and $v^{\Delta}(x)=w(x)$, we have

$$
\begin{aligned}
\int_{a}^{\infty} w(x) f^{q}(x) \Delta x= & \left.f^{q}(x) v(x)\right|_{a} ^{\infty} \\
& -\int_{a}^{\infty}\left[f^{q}(x)\right]^{\Delta} v^{\sigma}(x) \Delta x
\end{aligned}
$$

where $v(x)=-\int_{x}^{\infty} w(\tau) \Delta \tau$. Using the facts that $\lim _{x \rightarrow \infty} v(x)=0, f$ is bounded, and $f(a)=0$, we get

$$
\begin{gathered}
\int_{a}^{\infty} w(x) f^{q}(x) \Delta x=\int_{a}^{\infty}\left[-f^{q}(x)\right]^{\Delta} v^{\sigma}(x) \Delta x \\
=\int_{a}^{\infty}\left[f^{q}(x)\right]^{\Delta}\left(\int_{\sigma(x)}^{\infty} w(\tau) \Delta \tau\right) \Delta x .
\end{gathered}
$$

Since $f$ is increasing, we obtain

$$
\begin{aligned}
& \int_{a}^{\infty} w(x) f^{q}(x) \Delta x \\
& \quad \leq \int_{a}^{\infty}\left[f^{q}(x)\right]^{\Delta}\left(\int_{x}^{\infty} w(\tau) \Delta \tau\right) \Delta x .
\end{aligned}
$$

From (58) and (62), we see (note that $f$ is increasing) that

$$
\begin{aligned}
& \int_{a}^{\infty} w(x) f^{q}(x) \Delta x \\
& \quad \leq D^{q} \int_{a}^{\infty}\left[f^{q}(x)\right]^{\Delta}\left[\int_{x}^{\infty} h(\tau) \Delta \tau\right]^{q / p} \Delta x,
\end{aligned}
$$

and then

$$
\begin{aligned}
& \left(\int_{a}^{\infty} w(x) f^{q}(x) \Delta x\right)^{p / q} \\
& \quad \leq D^{p}\left(\int_{a}^{\infty}\left[f^{q}(x)\right]^{\Delta}\left[\int_{x}^{\infty} h(\tau) \Delta \tau\right]^{q / p} \Delta x\right)^{p / q} .
\end{aligned}
$$

Applying (36) with $\gamma=q / p, \alpha(x)=\left[f^{q}(x)\right]^{\Delta}$ and $\beta(\tau)=h(\tau)$ on the term

$$
\left(\int_{a}^{\infty}\left[f^{q}(x)\right]^{\Delta}\left[\int_{x}^{\infty} h(\tau) \Delta \tau\right]^{q / p} \Delta x\right)^{p / q},
$$

we have

$$
\begin{gathered}
\left(\int_{a}^{\infty}\left[f^{q}(x)\right]^{\Delta}\left[\int_{x}^{\infty} h(\tau) \Delta \tau\right]^{q / p} \Delta x\right)^{p / q} \\
\leq \int_{a}^{\infty} h(x)\left[\int_{a}^{\sigma(x)}\left[f^{q}(s)\right]^{\Delta} \Delta s\right]^{p / q} \Delta x .
\end{gathered}
$$

Substituting (66) into (64), we see (note that $f(a)=0$ ) that

$$
\begin{aligned}
& \left(\int_{a}^{\infty} w(x) f^{q}(x) \Delta x\right)^{p / q} \\
& \quad \leq D^{p} \int_{a}^{\infty} h(x)\left[\int_{a}^{\sigma(x)}\left[f^{q}(s)\right]^{\Delta} \Delta s\right]^{p / q} \Delta x \\
& \quad=D^{p} \int_{a}^{\infty} h(x)\left[f^{\sigma}(x)\right]^{p} \Delta x,
\end{aligned}
$$

and then

$$
\begin{aligned}
& \left(\int_{a}^{\infty} w(x) f^{q}(x) \Delta x\right)^{1 / q} \\
& \quad \leq D\left(\int_{a}^{\infty} h(x)\left[f^{\sigma}(x)\right]^{p} \Delta x\right)^{1 / p},
\end{aligned}
$$

which is the desired inequality (59). The proof is complete. 
Theorem 11. Assume that $\mathbb{T}$ is a time scale with, $b \in \mathbb{T}$. If $f$ is $C$-decreasing on $[a, b]_{\mathbb{T}}$ for $C \geq 1$ and $g$ is increasing on $[a, b]_{\mathbb{N}}$, such that $g(a)=0$, then

$$
\begin{aligned}
& \varphi\left(C \int_{a}^{b} f(x) g^{\Delta}(x) \Delta x\right) \\
& \quad \leq C \int_{a}^{b} f(x) g^{\Delta}(x) \varphi^{\prime}[f(x) g(x)] \Delta x .
\end{aligned}
$$

Proof. Denote

$$
\begin{aligned}
F(t):= & \varphi\left(C \int_{a}^{t} f(x) g^{\Delta}(x) \Delta x\right) \\
& -C \int_{a}^{t} f(x) g^{\Delta}(x) \varphi^{\prime}[f(x) g(x)] \Delta x,
\end{aligned}
$$

and

$$
G(t):=C \int_{a}^{t} f(x) g^{\Delta}(x) \Delta x .
$$

Therefore, we have from (70) and (71) that

$$
\begin{aligned}
F(t):= & \varphi(G(t)) \\
& -C \int_{a}^{t} f(x) g^{\Delta}(x) \varphi^{\prime}[f(x) g(x)] \Delta x .
\end{aligned}
$$

Since $f$ is $C$ - decreasing, then we have, for $t \geq x$, that $f(t) \leq$ $C f(x)$, and then we obtain (note $g$ is increasing and $g(a)=0$ ) that

$$
\begin{aligned}
\int_{a}^{t} C f(x) g^{\Delta}(x) \Delta x & \geq \int_{a}^{t} f(t) g^{\Delta}(x) \Delta x \\
& =f(t) \int_{a}^{t} g^{\Delta}(x) \Delta x \\
& =[g(t)-g(a)] f(t) \\
& =f(t) g(t) .
\end{aligned}
$$

Substituting (71) into (73), we have

$$
G(t) \geq f(t) g(t) .
$$

Applying the chain rule formula (13) on the term $\varphi(G(t))$, we see that there exists $\zeta \in[t, \sigma(t)]$, such that

$$
\varphi^{\Delta}(G(t))=\varphi^{\prime}(G(\zeta)) G^{\Delta}(t) .
$$

From (71), we obtain (note $g$ is increasing) that

$$
G^{\Delta}(t)=C f(t) g^{\Delta}(t) \geq 0,
$$

and then $G(t)$ is increasing on $[a, b]_{\mathbb{T}}$ and then we have, for $\zeta \geq t$, that

$$
G(\zeta) \geq G(t) .
$$

Since $\varphi$ is concave on $[0, \infty)$, then $\varphi^{\prime \prime}<0\left(\varphi^{\prime}\right.$ is decreasing on $[0, \infty)$ ) and, then, we observe from (77) that

$$
\varphi^{\prime}(G(\zeta)) \leq \varphi^{\prime}(G(t)) .
$$

Substituting (76) and (78) into (75), we get

$$
\varphi^{\Delta}(G(t)) \leq C f(t) g^{\Delta}(t) \varphi^{\prime}(G(t)) .
$$

From (74), we have that $\varphi^{\prime}(G(t)) \leq \varphi^{\prime}(f(t) g(t))$, and then we get (note $f$ is positive and $g$ is increasing) that

$$
C f(t) g^{\Delta}(t) \varphi^{\prime}(G(t)) \leq C f(t) g^{\Delta}(t) \varphi^{\prime}(f(t) g(t)),
$$

and thus we obtain from (79) that

$$
\varphi^{\Delta}(G(t)) \leq C f(t) g^{\Delta}(t) \varphi^{\prime}(f(t) g(t)) .
$$

From (72), we have

$$
F^{\Delta}(t)=\varphi^{\Delta}(G(t))-C f(t) g^{\Delta}(t) \varphi^{\prime}[f(t) g(t)] .
$$

Substituting (81) into (82), we see that $F^{\Delta}(t) \leq 0$, and therefore $F$ is decreasing on $[a, b]_{\mathbb{T}}$. Since $b>a$, we see that $F(b) \leq F(a)$. Since $\varphi(0)=0$, we have from (70) that

$$
F(a)=\varphi(0)=0,
$$

and then $F(b) \leq 0$, and then we have from (70), by sitting $t=b$, that

$$
\begin{aligned}
& \varphi\left(C \int_{a}^{b} f(x) g^{\Delta}(x) \Delta x\right) \\
& \quad \leq C \int_{a}^{b} f(x) g^{\Delta}(x) \varphi^{\prime}[f(x) g(x)] \Delta x,
\end{aligned}
$$

which is the desired inequality (69). The proof is complete.

Remark 12. As a special case of Theorem 11 when $\mathbb{T}=\mathbb{R}$, we get the integral inequality (6) proved by Pečarić et al. [2].

Remark 13. As a special case of Theorem 11 when $\mathbb{T}=\mathbb{R}, C=$ $1, \varphi(t)=t^{p}$, and $0<p \leq 1$, we obtain the integral inequality (1) proved by Heinig and Maligranda [1].

Remark 14. As a special case of Theorem 11 when $\mathbb{T}=\mathbb{N}$ and $a=1$, we see that the discrete inequality

$$
\varphi\left(C \sum_{k=1}^{N} f_{k} \Delta g_{k}\right) \leq C \sum_{k=1}^{N}\left(f_{k} \Delta g_{k}\right)\left(\varphi^{\prime}\left[f_{k} g_{k}\right]\right),
$$

holds for the $C$-decreasing sequence $f$ and the increasing sequence $g$ with $g(a)=0$.

Theorem 15. Assume that $\mathbb{T}$ is a time scale with, $b \in \mathbb{T}$. If $f$ is

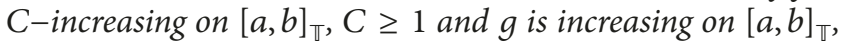
such that $g(a)=0$, then

$$
\begin{aligned}
& \varphi\left(\frac{1}{C} \int_{a}^{b} f(x) g^{\Delta}(x) \Delta x\right) \\
& \quad \geq \frac{1}{C} \int_{a}^{b} f(x) g^{\Delta}(x) \varphi^{\prime}\left[f^{\sigma}(x) g^{\sigma}(x)\right] \Delta x .
\end{aligned}
$$


Proof. Denote

$$
\begin{aligned}
F(t):= & \varphi\left(\frac{1}{C} \int_{a}^{t} f(x) g^{\Delta}(x) \Delta x\right) \\
& -\frac{1}{C} \int_{a}^{t} f(x) g^{\Delta}(x) \varphi^{\prime}\left[f^{\sigma}(x) g^{\sigma}(x)\right] \Delta x,
\end{aligned}
$$

and

$$
G(t):=\frac{1}{C} \int_{a}^{t} f(x) g^{\Delta}(x) \Delta x .
$$

Therefore, we have from (87) and (88) that

$$
\begin{aligned}
F(t)= & \varphi(G(t)) \\
& -\frac{1}{C} \int_{a}^{t} f(x) g^{\Delta}(x) \varphi^{\prime}\left[f^{\sigma}(x) g^{\sigma}(x)\right] \Delta x .
\end{aligned}
$$

Since $f$ is $C$ - increasing, then we have, for $x \leq \sigma(t)$, that $f(x) \leq C f^{\sigma}(t)$, and then we get (note $g$ is increasing and $g(a)=0)$ that

$$
\begin{aligned}
\int_{a}^{\sigma(t)} f(x) g^{\Delta}(x) \Delta x & \leq \int_{a}^{\sigma(t)} C f^{\sigma}(t) g^{\Delta}(x) \Delta x \\
& =C f^{\sigma}(t) \int_{a}^{\sigma(t)} g^{\Delta}(x) \Delta x \\
& =C f^{\sigma}(t)\left[g^{\sigma}(t)-g(a)\right] \\
& =C f^{\sigma}(t) g^{\sigma}(t),
\end{aligned}
$$

and thus

$$
\frac{1}{C} \int_{a}^{\sigma(t)} f(x) g^{\Delta}(x) \Delta x \leq f^{\sigma}(t) g^{\sigma}(t) .
$$

From (88), inequality (91) becomes

$$
G^{\sigma}(t) \leq f^{\sigma}(t) g^{\sigma}(t) .
$$

Applying the chain rule formula (13) on the term $\varphi(G(t))$, we see that there exists $\zeta \in[t, \sigma(t)]$, such that

$$
\varphi^{\Delta}(G(t))=\varphi^{\prime}(G(\zeta)) G^{\Delta}(t) .
$$

From (88), we obtain (note $g$ is increasing) that

$$
G^{\Delta}(t)=\frac{1}{C} f(t) g^{\Delta}(t) \geq 0
$$

then $G(t)$ is increasing on $[a, b]_{\mathbb{T}}$, and then we have, for $\zeta \leq$ $\sigma(t)$, that

$$
G(\zeta) \leq G^{\sigma}(t)
$$

Since $\varphi$ is concave on $[0, \infty)$, then $\varphi^{\prime \prime}<0\left(\varphi^{\prime}\right.$ is decreasing on $[0, \infty))$ and then we observe from $(95)$ that

$$
\varphi^{\prime}(G(\zeta)) \geq \varphi^{\prime}\left(G^{\sigma}(t)\right)
$$

Substituting (94) and (96) into (93), we get

$$
\varphi^{\Delta}(G(t)) \geq \frac{1}{C} f(t) g^{\Delta}(t) \varphi^{\prime}\left(G^{\sigma}(t)\right)
$$

From (92), we have that $\varphi^{\prime}\left(G^{\sigma}(t)\right) \geq \varphi^{\prime}\left(f^{\sigma}(t) g^{\sigma}(t)\right)$, and then we get (note $f$ is positive and $g$ is increasing) that

$$
\begin{aligned}
& \frac{1}{C} f(t) g^{\Delta}(t) \varphi^{\prime}\left(G^{\sigma}(t)\right) \\
& \quad \geq \frac{1}{C} f(t) g^{\Delta}(t) \varphi^{\prime}\left(f^{\sigma}(t) g^{\sigma}(t)\right) ;
\end{aligned}
$$

thus we obtain from (97) that

$$
\varphi^{\Delta}(G(t)) \geq \frac{1}{C} f(t) g^{\Delta}(t) \varphi^{\prime}\left(f^{\sigma}(t) g^{\sigma}(t)\right) .
$$

From (89), we have

$$
F^{\Delta}(t)=\varphi^{\Delta}(G(t))-\frac{1}{C} f(t) g^{\Delta}(t) \varphi^{\prime}\left[f^{\sigma}(t) g^{\sigma}(t)\right]
$$

Substituting (99) into (100), we see that $F^{\Delta}(t) \geq 0$, and therefore $F$ is increasing on $[a, b]_{\mathbb{T}}$. Since $b>a$, we see that $F(b) \geq F(a)$. Since $\varphi(0)=0$, we have from (87) that

$$
F(a)=\varphi(0)=0 ;
$$

then $F(b) \geq 0$, and then we have, from (87) by sitting $t=b$, that

$$
\begin{aligned}
& \varphi\left(\frac{1}{C} \int_{a}^{b} f(x) g^{\Delta}(x) \Delta x\right) \\
& \quad \geq \frac{1}{C} \int_{a}^{b} f(x) g^{\Delta}(x) \varphi^{\prime}\left[f^{\sigma}(x) g^{\sigma}(x)\right] \Delta x,
\end{aligned}
$$

which is the desired inequality (86). The proof is complete.

Remark 16. As a special case of Theorem 15 when $\mathbb{T}=\mathbb{R}$ and $\sigma(x)=x$, we get the integral inequality (7) proved by Pečarić et al. [2].

Remark 17. As a special case of Theorem 15 when $\mathbb{T}=\mathbb{N}$, $\sigma(n)=n+1$, and $a=1$, we see that the discrete inequality

$$
\varphi\left(\frac{1}{C} \sum_{k=1}^{N} f_{k} \Delta g_{k}\right) \geq \frac{1}{C} \sum_{k=1}^{N}\left(f_{k} \Delta g_{k}\right) \varphi^{\prime}\left[f_{k+1} g_{k+1}\right]
$$

holds for the $C$-increasing sequence $f$ and the increasing sequence $g$ with $g(a)=0$.

Theorem 18. Assume that $\mathbb{T}$ is a time scale with $a, b \in \mathbb{T}$. If $f$ is $C$-increasing on $[a, b]_{\mathbb{T}}, C \geq 1$, and $g$ is decreasing on $[a, b]_{\mathbb{T}}$, such that $g(b)=0$, then

$$
\begin{aligned}
& \varphi\left(C \int_{a}^{b} f(x)[-g(x)]^{\Delta} \Delta x\right) \\
& \quad \leq C \int_{a}^{b} f(x)[-g(x)]^{\Delta} \varphi^{\prime}\left[f^{\sigma}(x) g^{\sigma}(x)\right] \Delta x .
\end{aligned}
$$


Proof. Denote

$$
\begin{aligned}
F(t):= & -\varphi\left(C \int_{t}^{b} f(x)[-g(x)]^{\Delta} \Delta x\right) \\
& -C \int_{t}^{b} f(x) g^{\Delta}(x) \varphi^{\prime}\left[f^{\sigma}(x) g^{\sigma}(x)\right] \Delta x,
\end{aligned}
$$

and

$$
G^{*}(t):=C \int_{t}^{b} f(x)[-g(x)]^{\Delta} \Delta x .
$$

Therefore, we have from (105) and (106) that

$$
\begin{aligned}
F(t)= & -\varphi\left(G^{*}(t)\right) \\
& -C \int_{t}^{b} f(x) g^{\Delta}(x) \varphi^{\prime}\left[f^{\sigma}(x) g^{\sigma}(x)\right] \Delta x .
\end{aligned}
$$

Since $f$ is $C$ - increasing, then we have, for $\sigma(t) \leq x$, that $f^{\sigma}(t) \leq C f(x)$, and then we obtain (note $g$ is decreasing and $g(b)=0)$ that

$$
\begin{aligned}
& C \int_{\sigma(t)}^{b} f(x)[-g(x)]^{\Delta} \Delta x \\
& \quad \geq \int_{\sigma(t)}^{b} f^{\sigma}(t)[-g(x)]^{\Delta} \Delta x \\
& \quad=f^{\sigma}(t) \int_{\sigma(t)}^{b}[-g(x)]^{\Delta} \Delta x \\
& \quad=f^{\sigma}(t)\left[g^{\sigma}(t)-g(b)\right]=f^{\sigma}(t) g^{\sigma}(t) .
\end{aligned}
$$

Substituting (106) into (108), we observe that

$$
G^{*}(\sigma(t)) \geq f^{\sigma}(t) g^{\sigma}(t) .
$$

By applying the chain rule formula (13) on the term $\varphi\left(G^{*}(t)\right)$, we see that there exists $\zeta \in[t, \sigma(t)]$, such that

$$
\varphi^{\Delta}\left(G^{*}(t)\right)=\varphi^{\prime}\left(G^{*}(\zeta)\right)\left[G^{*}(t)\right]^{\Delta} .
$$

From (106), we obtain (note $g$ is decreasing) that

$$
\left[G^{*}(t)\right]^{\Delta}=C f(t) g^{\Delta}(t) \leq 0 ;
$$

then $G^{*}(t)$ is decreasing on $[a, b]_{\mathbb{T}}$, and then we have for $\zeta \leq$ $\sigma(t)$ that

$$
G^{*}(\zeta) \geq G^{*}(\sigma(t))
$$

Since $\varphi$ is concave on $[0, \infty)$, then $\varphi^{\prime \prime}<0\left(\varphi^{\prime}\right.$ is decreasing on $[0, \infty))$ and then we observe from (112) that

$$
\varphi^{\prime}\left(G^{*}(\zeta)\right) \leq \varphi^{\prime}\left(G^{*}(\sigma(t))\right) .
$$

Substituting (111) and (113) into (110), we get

$$
\varphi^{\Delta}\left(G^{*}(t)\right) \geq C f(t) g^{\Delta}(t) \varphi^{\prime}\left(G^{*}(\sigma(t))\right) .
$$

From (109), we have that $\varphi^{\prime}\left(G^{*}(\sigma(t))\right) \leq \varphi^{\prime}\left(f^{\sigma}(t) g^{\sigma}(t)\right)$, and then we get (note $f$ is positive and $g$ is decreasing) that

$$
\begin{aligned}
& C f(t) g^{\Delta}(t) \varphi^{\prime}\left(G^{*}(\sigma(t))\right) \\
& \quad \geq C f(t) g^{\Delta}(t) \varphi^{\prime}\left(f^{\sigma}(t) g^{\sigma}(t)\right),
\end{aligned}
$$

and thus we obtain from (114) that

$$
\varphi^{\Delta}\left(G^{*}(t)\right) \geq C f(t) g^{\Delta}(t) \varphi^{\prime}\left(f^{\sigma}(t) g^{\sigma}(t)\right),
$$

and then

$$
-\varphi^{\Delta}\left(G^{*}(t)\right) \leq-C f(t) g^{\Delta}(t) \varphi^{\prime}\left(f^{\sigma}(t) g^{\sigma}(t)\right) .
$$

From (107), we have

$$
\begin{aligned}
F^{\Delta}(t)= & -\varphi^{\Delta}\left(G^{*}(t)\right) \\
& +C f(t) g^{\Delta}(t) \varphi^{\prime}\left[f^{\sigma}(t) g^{\sigma}(t)\right] .
\end{aligned}
$$

Substituting (117) into (118), we see that $F^{\Delta}(t) \leq 0$, and therefore $F$ is decreasing on $[a, b]_{\mathbb{T}}$. Since $b>a$, we see that $F(b) \leq F(a)$. Since $\varphi(0)=0$, we have from (105) that

$$
F(b)=-\varphi(0)=0 ;
$$

then $F(a) \geq 0$, and then we have, from (105) by sitting $t=a$, that

$$
\begin{aligned}
& \varphi\left(C \int_{a}^{b} f(x)[-g(x)]^{\Delta} \Delta x\right) \\
& \quad \leq C \int_{a}^{b} f(x)[-g(x)]^{\Delta} \varphi^{\prime}\left[f^{\sigma}(x) g^{\sigma}(x)\right] \Delta x,
\end{aligned}
$$

which is the desired inequality (104). The proof is complete.

Remark 19. As a special case of Theorem 18 when $\mathbb{T}=\mathbb{R}$ and $\sigma(x)=x$, we get the integral inequality (8) proved by Pečarić et al. [2].

Remark 20. As a special case of Theorem 18 when $\mathbb{T}=\mathbb{R}$, $\sigma(n)=n, C=1, \varphi(t)=t^{p}$, and $0<p \leq 1$, we obtain the integral inequality (2) proved by Heinig and Maligranda [1].

Remark 21. As a special case of Theorem 18 when $\mathbb{T}=\mathbb{N}$, $\sigma(n)=n+1$, and $a=1$, we see that the discrete inequality

$$
\begin{aligned}
& \varphi\left(C \sum_{k=1}^{N} f_{k} \Delta\left[-g_{k}\right]\right) \\
& \leq C \sum_{k=1}^{N}\left(f_{k} \Delta\left[-g_{k}\right]\right) \varphi^{\prime}\left[f_{k+1} g_{k+1}\right],
\end{aligned}
$$

holds for the $C$-increasing sequence $f$ and the decreasing sequence $g$ with $g(b)=0$. 
Theorem 22. Assume that $\mathbb{T}$ is a time scale with $a, b \in \mathbb{T}$. If $f$ is $C$-decreasing on $[a, b]_{\mathbb{T}}, C \geq 1$, and $g$ is decreasing on $[a, b]_{\mathbb{T}}$, such that $g(b)=0$, then

$$
\begin{aligned}
& \varphi\left(\frac{1}{C} \int_{a}^{b} f(x)[-g(x)]^{\Delta} \Delta x\right) \\
& \quad \geq \frac{1}{C} \int_{a}^{b} f(x)[-g(x)]^{\Delta} \varphi^{\prime}[f(x) g(x)] \Delta x .
\end{aligned}
$$

Proof. Denote

$$
\begin{aligned}
F(t):= & -\varphi\left(\frac{1}{C} \int_{t}^{b} f(x)[-g(x)]^{\Delta} \Delta x\right) \\
& +\frac{1}{C} \int_{t}^{b} f(x)[-g(x)]^{\Delta} \varphi^{\prime}[f(x) g(x)] \Delta x,
\end{aligned}
$$

and

$$
G^{*}(t):=\frac{1}{C} \int_{t}^{b} f(x)[-g(x)]^{\Delta} \Delta x .
$$

Therefore, we have from (123) and (124) that

$$
\begin{aligned}
F(t)= & -\varphi\left(G^{*}(t)\right) \\
& +\frac{1}{C} \int_{t}^{b} f(x)[-g(x)]^{\Delta} \varphi^{\prime}[f(x) g(x)] \Delta x .
\end{aligned}
$$

Since $f$ is $C$-decreasing, then we have, for $x \geq t$, that $f(x) \leq$ $C f(t)$, and then we obtain (note $g$ is decreasing and $g(b)=0$ ) that

$$
\begin{aligned}
& \int_{t}^{b} f(x)[-g(x)]^{\Delta} \Delta x \leq \int_{t}^{b} C f(t)[-g(x)]^{\Delta} \Delta x \\
& \quad=C f(t) \int_{t}^{b}[-g(x)]^{\Delta} \Delta x=C f(t)[g(t)-g(b)] \\
& \quad=C f(t) g(t),
\end{aligned}
$$

and then

$$
\frac{1}{C} \int_{t}^{b} f(x)[-g(x)]^{\Delta} \Delta x \leq f(t) g(t) .
$$

Substituting (124) into (127), we get

$$
G^{*}(t) \leq f(t) g(t) .
$$

By applying the chain rule formula (13) on the term $\varphi\left(G^{*}(t)\right)$, we see that there exists $d \in[t, \sigma(t)]$, such that

$$
\varphi^{\Delta}\left(G^{*}(t)\right)=\varphi^{\prime}\left(G^{*}(d)\right)\left[G^{*}(t)\right]^{\Delta} .
$$

From (124), we obtain (note $g$ is decreasing) that

$$
\left[G^{*}(t)\right]^{\Delta}=\frac{1}{C} f(t) g^{\Delta}(t) \leq 0 ;
$$

then $G^{*}(t)$ is decreasing on $[a, b]_{\mathbb{T}}$, and then we have, for $d \geq$ $t$, that

$$
G^{*}(d) \leq G^{*}(t)
$$

Since $\varphi$ is concave on $[0, \infty)$, then $\varphi^{\prime \prime}<0\left(\varphi^{\prime}\right.$ is decreasing on $[0, \infty))$ and then, we observe from (131) that

$$
\varphi^{\prime}\left(G^{*}(d)\right) \geq \varphi^{\prime}\left(G^{*}(t) .\right.
$$

Substituting (130) and (132) into (129), we get

$$
\varphi^{\Delta}\left(G^{*}(t)\right) \leq \frac{1}{C} f(t) g^{\Delta}(t) \varphi^{\prime}\left(G^{*}(t)\right.
$$

From (128), we have that $\varphi^{\prime}\left(G^{*}(t)\right) \geq \varphi^{\prime}(f(t) g(t))$, and then we get (note $f$ is positive and $g$ is decreasing) that

$$
\begin{aligned}
& \frac{1}{C} f(t) g^{\Delta}(t) \varphi^{\prime}\left(G^{*}(t)\right) \\
& \quad \leq \frac{1}{C} f(t) g^{\Delta}(t) \varphi^{\prime}(f(t) g(t))
\end{aligned}
$$

thus we obtain from (133) that

$$
\varphi^{\Delta}\left(G^{*}(t)\right) \leq \frac{1}{C} f(t) g^{\Delta}(t) \varphi^{\prime}(f(t) g(t)),
$$

and then

$$
-\varphi^{\Delta}\left(G^{*}(t)\right) \geq-\frac{1}{C} f(t) g^{\Delta}(t) \varphi^{\prime}(f(t) g(t)) .
$$

From (125), we have

$$
\begin{aligned}
F^{\Delta}(t)= & -\varphi^{\Delta}\left(G^{*}(t)\right) \\
& +\frac{1}{C} f(t) g^{\Delta}(t) \varphi^{\prime}[f(t) g(t)] .
\end{aligned}
$$

Substituting (136) into (137), we see that $F^{\Delta}(t) \geq 0$, and therefore $F$ is increasing on $[a, b]_{\mathbb{T}}$. Since $b>a$, we see that $F(b) \geq F(a)$. Since $\varphi(0)=0$, we have from (123) that

$$
F(b)=-\varphi(0)=0
$$

then $F(a) \leq 0$, and then we have, from (123) by sitting $t=a$, that

$$
\begin{aligned}
& -\varphi\left(\frac{1}{C} \int_{a}^{b} f(x)[-g(x)]^{\Delta} \Delta x\right) \\
& \quad+\frac{1}{C} \int_{a}^{b} f(x)[-g(x)]^{\Delta} \varphi^{\prime}[f(x) g(x)] \Delta x \\
& \leq 0,
\end{aligned}
$$

and thus

$$
\begin{aligned}
& \varphi\left(\frac{1}{C} \int_{a}^{b} f(x)[-g(x)]^{\Delta} \Delta x\right) \\
& \geq \frac{1}{C} \int_{a}^{b} f(x)[-g(x)]^{\Delta} \varphi^{\prime}[f(x) g(x)] \Delta x
\end{aligned}
$$

which is the desired inequality (122). The proof is complete. 
Remark 23. As a special case of Theorem 22 when $\mathbb{T}=\mathbb{R}$, we get the integral inequality (9) proved by Pečarić et al. [2].

Remark 24. As a special case of Theorem 22 when $\mathbb{T}=\mathbb{N}$ and $a=1$, we see that the discrete inequality

$$
\varphi\left(\frac{1}{C} \sum_{k=1}^{N} f_{k} \Delta\left[-g_{k}\right]\right) \geq \frac{1}{C} \sum_{k=1}^{N} f_{k} \Delta\left[-g_{k}\right] \varphi^{\prime}\left[f_{k} g_{k}\right]
$$

holds for the $C$-decreasing sequence $f$ and the decreasing sequence $g$ with $g(b)=0$.

\section{Data Availability}

The data used to support the findings of this study are included within the article.

\section{Conflicts of Interest}

The authors declare that they have no conflicts of interest.

\section{References}

[1] H. Heinig and L. Maligranda, "Weighted inequalities for monotone and concave functions," Studia Mathematica, vol. 116, no. 2, pp. 133-165, 1995.

[2] J. Pecari, I. Peri, and L. E. Persson, "Integral inequalities for monotone functions," Journal of Mathematical Analysis and Applications, vol. 215, no. 1, pp. 235-251, 1997.

[3] R. P. Agarwal, D. O’Regan, and S. H. Saker, Dynamic Inequalities on Time Scales, Springer, 2014.

[4] R. P. Agarwal, D. O’Regan, and S. H. Saker, Hardy Type Inequalities on Time Scales, Springer, 2016.

[5] M. Bohner and A. Peterson, Dynamic Equations on Time Scales: An Introduction with Applications, Birkhauser, Boston, Massachusetts, Mass, USA, 2001.

[6] R. Bibi, M. Bohner, J. Pecaric, and S. Varosanec, "Minkowski and Beckenbach-Dresher inequalities and functionals on time scales," Journal of Mathematical Inequalities, vol. 7, no. 3, pp. 299-312, 2013.

[7] M. Bohner and S. G. Georgiev, "Multiple integration on time scales," in Multivariable Dynamic Calculus on Time Scales, pp. 449-515, Springer, Cham, Switzerland, 2016.

[8] J. A. Oguntuase and L.-E. Persson, "Time scales Hardy-type inequalities via superquadracity," Annals of Functional Analysis, vol. 5, no. 2, pp. 61-73, 2014.

[9] P. Rehak, "Hardy inequality on time scales and its application to half-linear dynamic equations," Journal of Inequalities and Applications, vol. 2005, no. 5, pp. 495-507, 2005.

[10] S. H. Saker, R. R. Mahmoud, and A. Peterson, "Weighted Hardytype inequalities on time scales with applications," Mediterranean Journal of Mathematics, vol. 13, no. 2, pp. 585-606, 2016.

[11] S. H. Saker and D. O’Regan, "Hardy and Littlewood inequalities on time scales," Bulletin of the Malaysian Mathematical Sciences Society, vol. 39, no. 2, pp. 527-543, 2016.

[12] M. Bohner and A. Peterson, Advances in Dynamic Equations on Time Scales, Springer Science and Business Media, 2002. 


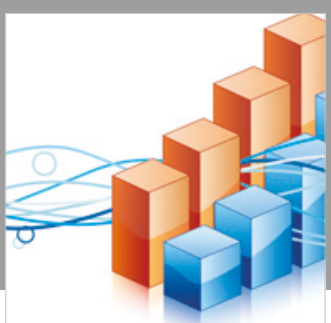

Advances in

Operations Research

\section{-n-m}
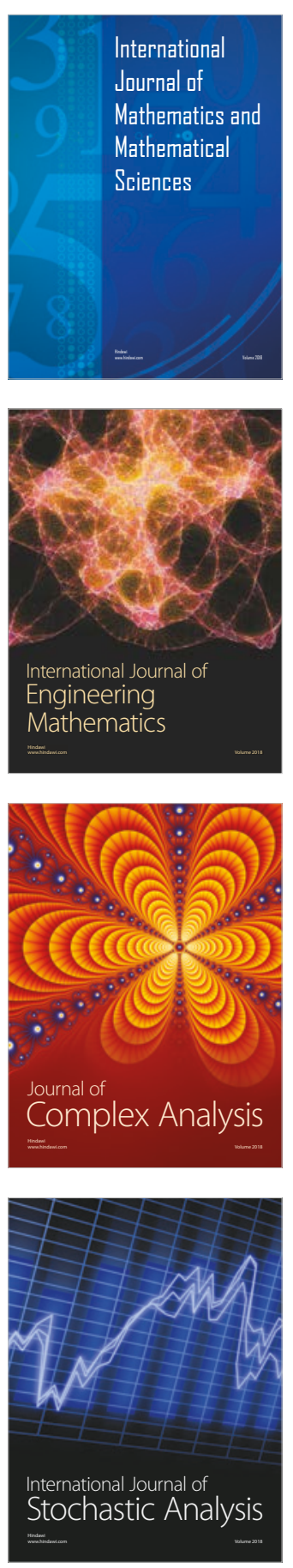
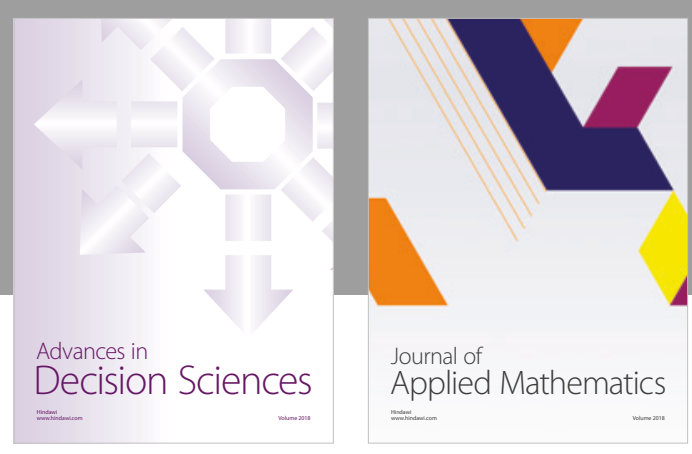

Journal of

Applied Mathematics
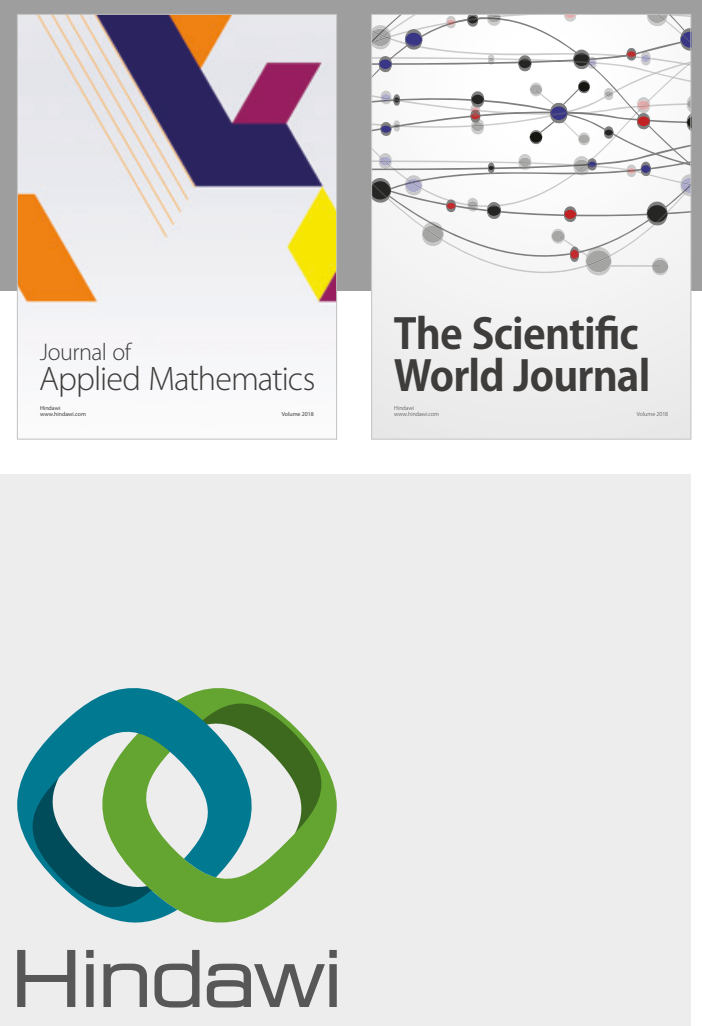

Submit your manuscripts at

www.hindawi.com

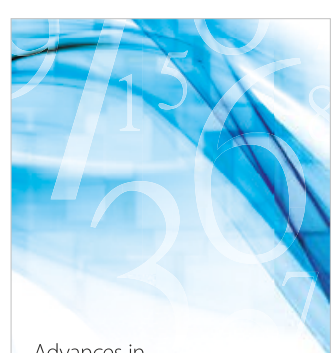

Advances in
Numerical Analysis
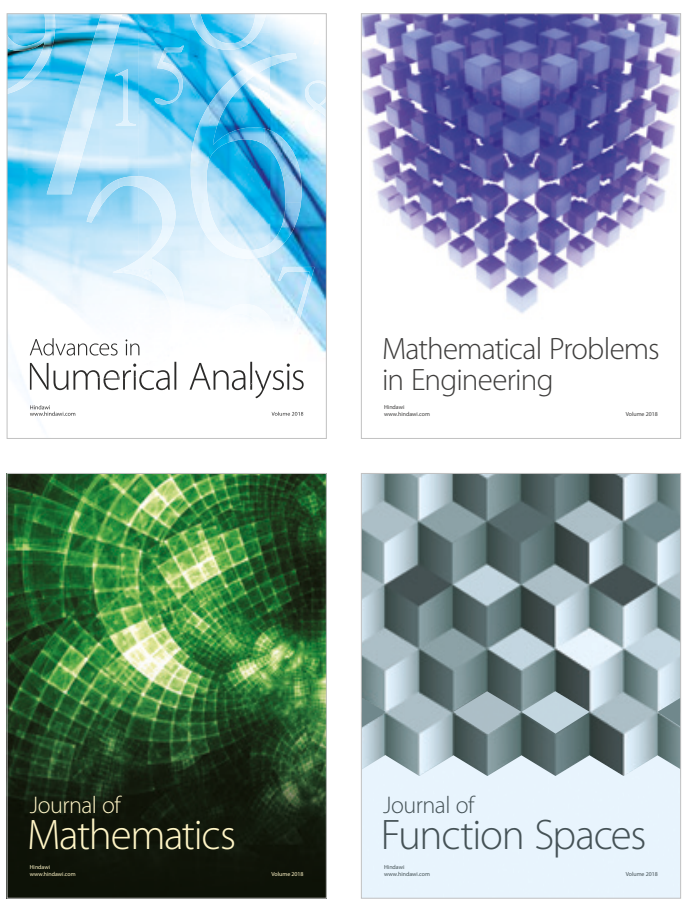

Mathematical Problems in Engineering

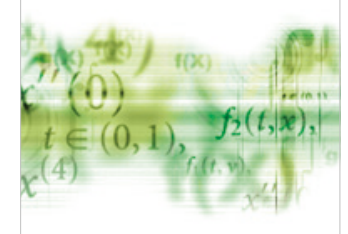

International Journal of

Differential Equations

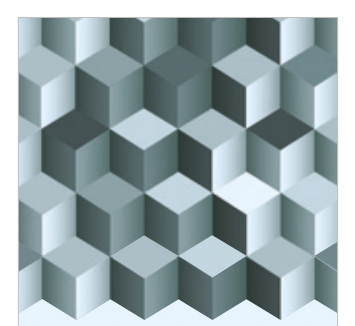

Journal of

Function Spaces

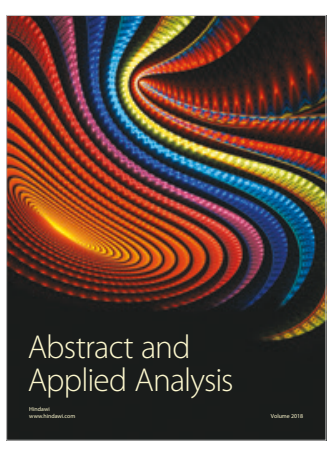

The Scientific

World Journal

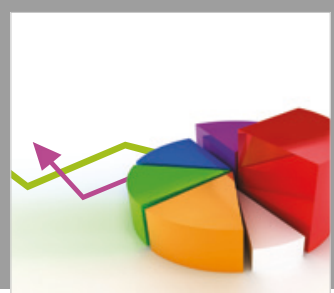

Journal of

Probability and Statistics
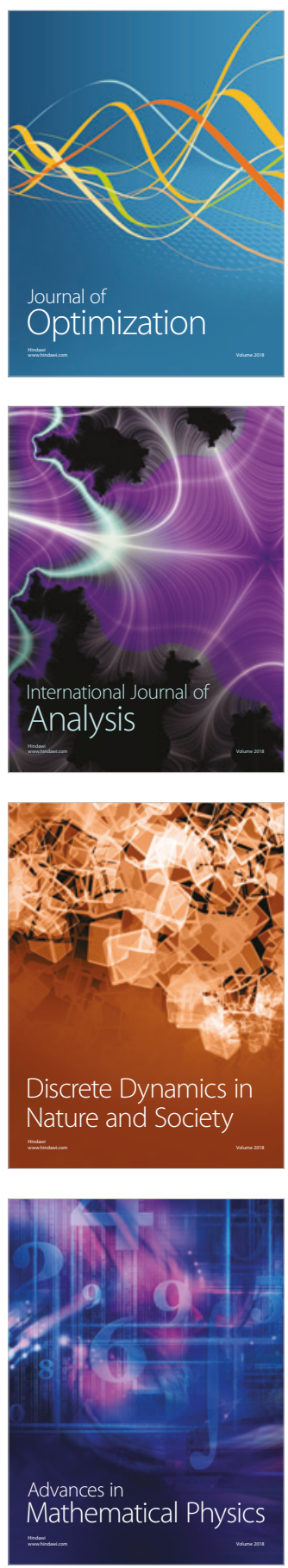